\section{CASE REPORT}

H.E. Möller

T. Mildner

C. Preul

C. Zimmer

D. Yves von Cramon

\title{
Assessment of Collateral Supply by Two-Coil Continuous Arterial Spin Labeling after Coil Occlusion of the Internal Carotid Artery
}

\begin{abstract}
SUMmARY: A patient undergoing coil occlusion of a left internal carotid artery aneurysm was investigated by continuous arterial spin labeling MR imaging to evaluate perfusion territory mapping. Labeling was restricted to the left- or right-sided carotid artery by use of a separate neck coil. Before embolization, perfusion contrast was largely restricted to the labeled hemisphere. After embolization, perfusion contrast was created symmetrically in both hemispheres on labeling the right side, verifying sufficient collateral supply.
\end{abstract}

I n carotid artery occlusive disease, the degree of stenosis is a critical parameter in the potential development of stroke. ${ }^{1}$ Knowledge of the amount of compromise of cerebral blood flow $(\mathrm{CBF})$, of the affected perfusion territories, and of the status of collaterals would additionally be desirable for assessing the hemodynamic status of the brain and predicting the outcome. Perfusion MR imaging is increasingly being used for quantifying $\mathrm{CBF}$ and assessing cerebrovascular function. A totally noninvasive technique is arterial spin labeling, which exploits magnetically labeled arterial water as an endogenous, diffusible tracer to quantify perfusion from 2 sets of images obtained with labeling and a control condition. Approaches for mapping the flow territories of major brain-supplying arteries have also been suggested. ${ }^{2-6}$ For this study, we used continuous arterial spin labeling (CASL) with a local transmit coil at the neck for selectively labeling the blood of either the right or left carotid artery and a separate head coil for imaging. ${ }^{2}$ The goal was to evaluate the potential of perfusion territory imaging for an assessment of the hemodynamic status of the brain in a patient undergoing coil occlusion of the internal carotid artery (ICA).

\section{Case Report}

In a male 28-year-old patient, a dissecting aneurysm of the left ICA had developed after an accident during childhood and caused a minor stroke. Carotid balloon occlusion test revealed that the patient would tolerate permanent ICA occlusion because of sufficient collateral supply to the left hemisphere. Multiple Guglielmi detachable coils (Boston Scientific, Natick, Mass) were then inserted for the occlusion of the distal ICA aneurysm through a microcatheter. Postembolization angiograms verified total occlusion of the left ICA.

After obtaining informed written consent, CASL was performed on a 3T MedSpec 30/100 scanner (Bruker, Ettlingen, Germany), 1 day before and 3 months after embolization. A 6-cm-diameter surface coil

Received October 13, 2006; accepted after revision November 27.

From the Max Planck Institute for Human Cognitive and Brain Sciences (H.E.M., T.M., C.P., D.Y.v.C.), Leipzig, Germany; Department of Neurology (C.P.), University Hospital Jena, Jena, Germany; Department of Diagnostic Radiology (C.Z.), University Hospital Leipzig, Leipzig, Germany; Department of Neuroradiology (C.Z.), University Hospital Rechts der Isar, Munich, Germany.

H.E.M. and T.M. contributed equally to this work.

Paper previously presented at: Annual Scientific Meeting of the International Society for Magnetic Resonance in Medicine, May 6-12, 2006; Seattle, Wash.

Address correspondence to Harald E. Möller, Max Planck Institute for Human Cognitive and Brain Sciences, Stephanstraße 1A, 04103 Leipzig, Germany; e-mail: moeller@cbs.mpg.de DOI 10.3174/ajnr.A0552 was centered in the region of the right carotid bifurcation, and radiofrequency irradiation $(1.2 \mathrm{~W})$ and a magnetic field gradient $(2.5$ $\mathrm{mT} / \mathrm{m}$ ) along the z-direction were applied during labeling periods of $\tau=3.5$ seconds. Estimates of the labeling efficiency yielded $\alpha=85 \% .{ }^{7}$ One-hundred repetitions (TR, 7 seconds) were acquired with and without application of CASL during odd and even repetitions, respectively. To account for the finite transit time from the labeling plane to the region of interest, a postlabeling delay of $w=1.5$ seconds was introduced before image acquisition. Eight axial sections (thickness, 5 $\mathrm{mm}$; intersection distance, $6 \mathrm{~mm}$ ) were recorded sequentially from superior to inferior with spin-echo echo-planar imaging (TE, $50 \mathrm{~ms}$; bandwidth, $100 \mathrm{kHz}$; matrix, $64 \times 64$; FOV, $192 \mathrm{~mm}$ ) and a quadrature helmet coil. ${ }^{8} \mathrm{~T} 1$-weighted images were recorded at the identical section positions as anatomic reference. In the pre-embolization examination, the whole procedure was repeated after repositioning the label coil for application of CASL on the left side.

All of the perfusion maps were registered to a $3 \mathrm{D}$ dataset acquired in a separate session for a direct comparison of individual sections. Images measured during the CASL and control conditions were averaged separately to create a difference map. Estimates of CBF were obtained from the signal intensity change, $\bar{S}_{C A S L}-\bar{S}_{c t r l}$, according to the following?

$$
\begin{gathered}
\frac{\bar{S}_{C A S L}-\bar{S}_{c t r l}}{\bar{S}_{c t r l}}=-2 \alpha \frac{C B F}{\lambda} T_{1 a p p} e^{-w / T_{1 a p p}}\left(1-e^{-\tau / T_{1 a p p}}\right) \\
\text { with } \frac{1}{\mathrm{~T}_{1 a p p}}=\frac{1}{\mathrm{~T}_{1 b}}+\frac{C B F}{\lambda}
\end{gathered}
$$

In this equation, a blood-brain partition coefficient $\lambda$ is $0.9 \mathrm{~mL} / \mathrm{g}$, and a longitudinal relaxation time $T_{1 b}$ is 1.3 seconds for cortical gray matter (GM). $\bar{S}_{\text {CASL }}$ and $\bar{S}_{c t r l}$ are averaged signal intensity amplitudes obtained with and without application of CASL, respectively, and $T_{\text {lapp }}$ is the apparent longitudinal relaxation time.

Quantitative perfusion maps obtained before left ICA occlusion are shown in Fig $1 A, B$. With left-sided CASL, perfusion-related signal intensity changes were found only in leptomeningeal branches of the left middle cerebral artery (MCA) flow territory (Fig $1 B$, red arrow), whereas with right-sided CASL, signal intensity changes were present in the flow territory of the right MCA (Fig $1 A$, red arrow) and also in the territories of leptomeningeal branches of both the right and left anterior cerebral artery (ACA) surrounding the interhemispheric fissure (Fig $1 B$, blue arrow). Figure $1 C$ shows maps recorded with rightsided CASL after left ICA occlusion. Perfusion contrast was now created in both hemispheres in flow territories of the right and left ACA (Fig 1C, blue arrow), as well as that of the right and left MCA (Fig 1C, 

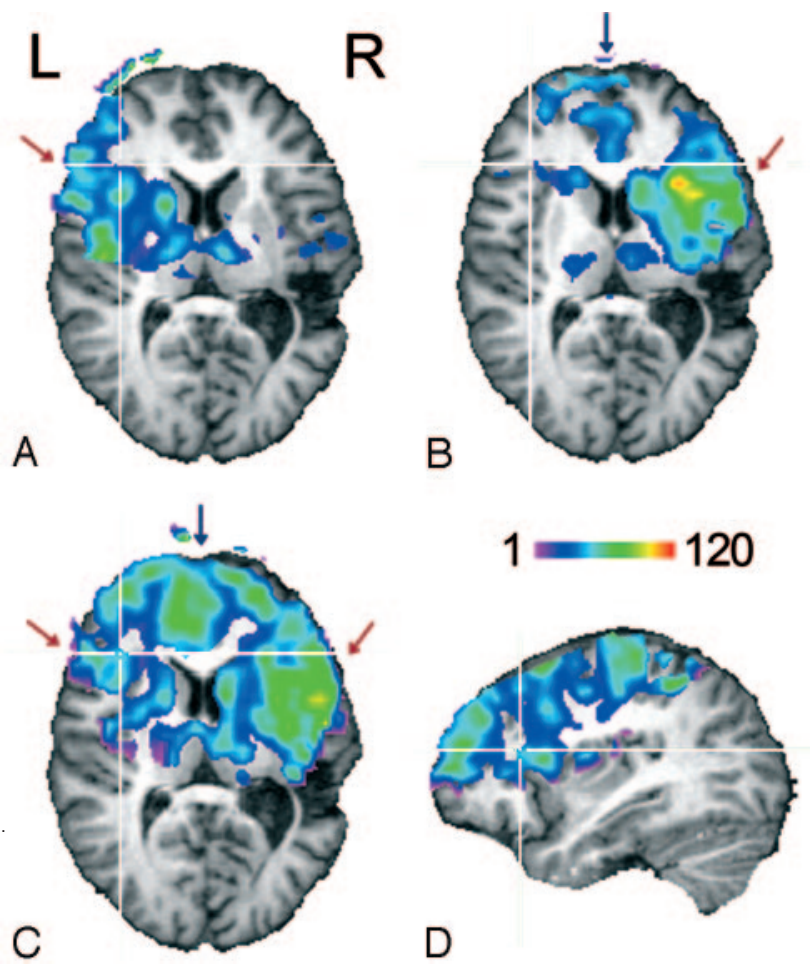

Fig 1. Perfusion territories of the $(A)$ left and $(B)$ right ICA recorded during the first examination and of the $(C)$ right ICA recorded 3 months after coil occlusion of the left ICA. The color code indicates CBF values in milliliters per minute per 100 grams. Only voxels with a significant signal intensity change between label and no-label conditions (thresholded at $P<.05$ ) are displayed.

$D$, The coverage of the section package can be assessed from the sagittal section (left hemisphere).

\begin{tabular}{lcr}
\hline \multicolumn{2}{l}{ Gray Matter CBF values of the ICA flow territories } \\
\hline Variable & $\begin{array}{c}\text { Left } \\
\text { Hemisphere }\end{array}$ & $\begin{array}{c}\text { Right } \\
\text { Hemisphere }\end{array}$ \\
\hline Before left ICA occlusion & $43.5 \pm 3.0$ & $48.9 \pm 4.8$ \\
After left ICA occlusion & $51.5 \pm 7.1$ & $54.1 \pm 8.5$ \\
\hline
\end{tabular}

Note:-Data are mean \pm SD in milliliters per minute per $100 \mathrm{~g}$. CBF indicates cerebral blood flow; ICA, internal carotid artery.

red arrow). CBF values averaged separately over the left and right hemisphere are summarized in the Table.

\section{Discussion}

The pre-embolization results demonstrate that the flow territories of the left and right ICA can be delineated selectively with the 2-coil CASL technique. With the current setup, coverage in deeper brain structures (eg, the inferior parietal lobe) was limited by the sensitive volume of the helmet coil (length, $13 \mathrm{~cm}$ ) and the angulation of the section package (Fig 1D). Improvement is straightforward by use of an appropriately modified imaging coil.

The distribution of perfusion contrast over both hemispheres after left ICA occlusion, which was observed on unilateral, right-sided CASL, verifies the existence of sufficient collateral supply, presumably via the circle of Willis as the primary pathway. This finding indicating a high sensitivity of the CASL technique agrees well with the result from the preceding carotid balloon occlusion test. Other than such qualitative correspondence, quantitative assessment of the ade- quacy of collaterals is obtained from the data in the table demonstrating equal postembolization levels of GM perfusion in both hemispheres within the experimental accuracy. The averaged CBF of GM in the unaffected right hemisphere and its variation by $10 \%$ between successive examinations are similar to values observed in a previous scan/rescan CASL study in normal volunteers. ${ }^{10}$ The data recorded before embolization in the flow territory of the left ICA seem to indicate a CBF reduction of borderline significance $(P=.09$ obtained from a section-by-section comparison and paired-samples $t$ test). Alternatively, a prolonged transit time, which may be attributed to the presence of the aneurysm, could also lead to artificially reduced CBF values. Recently, $\delta=1930 \pm 110$ ms was measured for the time the arterial water needs to travel from the labeling plane at the neck to the tissue in the ICA flow territory. ${ }^{11}$ A quantitative analysis within the framework of the 2-compartment model (compare equation 1 from Wang et $\mathrm{al}^{12}$ ) indicates that a transit time increase by $200 \mathrm{~ms}$ leads to a reduction of the signal intensity change by $13 \%$, which would explain the observed interhemispheric difference.

\section{Conclusion}

Two-coil CASL has a potential to assess the significance of carotid artery stenosis and compensating collateral flow. Because of the possibility to perform repeated studies, it may be suited for screening purposes and for monitoring perfusion changes after therapeutic interventions, which is not adequately achieved by more invasive methods. A 10\% range of normal variation and potentially confounding transit time effects impose limitations for detecting subtle CBF changes and should be addressed in further investigations.

\section{References}

1. Executive Committee for the Asymptomatic Carotid Atherosclerosis Study. Endarterectomy for asymptomatic carotid artery stenosis. JAMA 1995;273:1421-28

2. Zaharchuk G, Ledden PJ, Kwong KK, et al. Multislice perfusion and perfusion territory imaging in humans with separate label and image coils. Magn Reson Med 1999;41:1093-98

3. Trampel R, Mildner T, Goerke U, et al. Continuous arterial spin labeling using a local magnetic field gradient coil. Magn Reson Med 2002;48:543-46

4. Davies NP, Jezzard P. Selective arterial spin labeling (SASL): perfusion territory mapping of selected feeding arteries tagged using two-dimensional radiofrequency pulses. Magn Reson Med 2003;49:1133-42

5. Werner R, Alfke K, Schaeffter T, et al. Brain perfusion territory imaging applying oblique-plane arterial spin labeling with a standard send/receive head coil. Magn Reson Med 2004;52:1443-47

6. Hendrikse J, van der Grond J, Lu H, et al. Flow territory mapping of the cerebral arteries with regional perfusion MRI. Stroke 2004;35:882-87

7. Trampel R, Jochimsen TH, Mildner T, et al. Efficiency of flow-driven adiabatic spin inversion under realistic experimental conditions: a computer simulation. Magn Reson Med 2004;51:1187-93

8. Driesel W, Merkle H, Hetzer S, et al. Reengineered helmet coil for human brain imaging. Concepts Magn Reson B (Magn Reson Eng) 2005;27B:64-74

9. Mildner T, Trampel R, Möller HE, et al. Functional perfusion imaging using continuous arterial spin labeling with separate labeling and imaging coils at 3 T. Magn Reson Med 2003;49:791-95

10. Floyd TF, Ratcliffe SJ, Wang J, et al. Precision of the CASL-perfusion MRI technique for the measurement of cerebral blood flow in whole brain and vascular territories. J Magn Reson Imaging 2003;18:649-55

11. Mildner T, Möller HE, Driesel W, et al. Continuous arterial spin labeling at the human common carotid artery: the influence of transit times. NMR Biomed 2005; $18: 19-23$

12. Wang J, Alsop DC, Li L, et al. Comparison of quantitative perfusion imaging using arterial spin labeling at $\mathbf{1 . 5}$ and $\mathbf{4 . 0}$ tesla. Magn Reson Med 2002;48: 242-54 